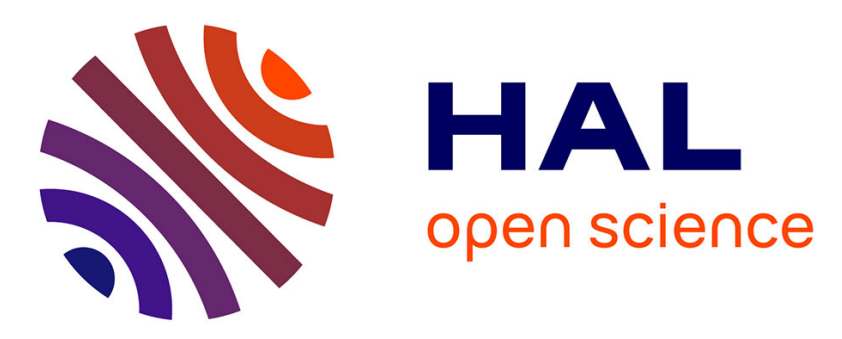

\title{
Quantification of natural products in herbal supplements: a combined NMR approach applied on goldenseal
}

Phuong Mai Le, Clément Milande, Estelle Martineau, Patrick Giraudeau, Jonathan Farjon

\section{To cite this version:}

Phuong Mai Le, Clément Milande, Estelle Martineau, Patrick Giraudeau, Jonathan Farjon. Quantification of natural products in herbal supplements: a combined NMR approach applied on goldenseal. Journal of Pharmaceutical and Biomedical Analysis, 2019, 165, pp.155-161. 10.1016/j.jpba.2018.11.062 . hal-03130979

\section{HAL Id: hal-03130979 \\ https://hal.science/hal-03130979}

Submitted on 4 Feb 2021

HAL is a multi-disciplinary open access archive for the deposit and dissemination of scientific research documents, whether they are published or not. The documents may come from teaching and research institutions in France or abroad, or from public or private research centers.
L'archive ouverte pluridisciplinaire HAL, est destinée au dépôt et à la diffusion de documents scientifiques de niveau recherche, publiés ou non, émanant des établissements d'enseignement et de recherche français ou étrangers, des laboratoires publics ou privés. 


\section{Quantification of natural products in herbal supplements: a combined NMR approach applied on goldenseal}

Phuong Mai Le ${ }^{\mathrm{a} *}$, Clément Milande ${ }^{\mathrm{b}}$, Estelle Martineau ${ }^{\mathrm{b}, \mathrm{c}}$, Patrick Giraudeau ${ }^{\mathrm{b}, \mathrm{d}}$ and Jonathan Farjon $^{\mathrm{b}, *}$

a Metrology, National Research Council Canada, 1200 Montreal road, Ottawa, ON K1A, OR6, Canada

$b$ EBSI Team, Chimie et Interdisciplinarité: Synthèse, Analyse, Modélisation (CEISAM), Université de Nantes, CNRS, UMR 6230, BP 92208 - 2, rue de la Houssinière, 44322 Nantes, France

c SpectroMaitrise, CAPACITÉS SAS, 26 Bd Vincent Gâche, 44200 Nantes, France

d Institut Universitaire de France, 1 rue Descartes, 75005 Paris Cedex 05, France

Corresponding authors:

Phuong Mai Le

PhuongMai.Le@nrc-cnrc.gc.ca

Phone 16139919406

Jonathan Farjon

Jonathan.Farjon@univ-nantes.fr

Phone 33251125434 


\section{Abstract}

2 Authentication of natural products is of major relevance in the context of manufactured drugs or

3 herbal supplements since such active products generate a lucrative market. The analytical method

4 to identify and quantify valuable natural products is critical for quality control and product

5 assignment of herbal supplements. In this framework, we propose to apply a recently developed

6 quantitative 2D NMR approach called Q QUIPU (Quick QUantItative Perfected and pUre shifted)

7 in combination with $1 \mathrm{D}{ }^{1} \mathrm{H}$ NMR capable to access the concentration of three major alkaloids,

8 berberine, hydrastine and canadine, in the root extract of goldenseal (Hydrastis canadensis), one

9 of the 20 most popular herbal supplements used worldwide. We highlight the complementarity of

$101 \mathrm{D}$ and 2D quantitative NMR to accurately assess the amount of alkaloids with different range of

11 concentrations and stability within extracts. In particular, unstable natural products having non-

12 overlapped signals like berberine could only be quantified by sensitive and fast $1 \mathrm{D}{ }^{1} \mathrm{H}$, while

13 overlapped signals of hydrastine and low intense ones of canadine could only be quantified with

14 the recent 2D Q QUIPU HSQC.

15 Results obtained from this combined approach have led to a good accuracy $(<10 \%)$ as compared 16 with coupled UPLC-MS/UV techniques. This quantitative NMR approach paves the way to

17 numerous applications where the accurate quantification of targeted compounds in complex

18 mixtures is required, for instance in agricultural, food and pharmaceuticals products.

21 Keywords: Herbal supplements, Goldenseal (Hydrastine canadensis), alkaloids, Quantitative 22 NMR, 1D ${ }^{1} \mathrm{H}$ and 2D Q QUIPU HSQC 


\section{Introduction}

Counterfeit and/or illegally manufactured drugs as well as herbal medicines have become a

27 worldwide public health issue. Herbal supplements are increasingly used as food or dietary

28 supplements and therefore have become a huge lucrative market. For their perceived benefits,

29 botanical extracts are key ingredients in nutraceuticals and personal care products. Their quality

30 depends on the quantity of active key components and on the absence of undesired materials such

31 as adulterants and residual solvents. The beneficial claims depend on the presence specific

32 molecules in the extracts, which therefore need to be identified and accurately quantified.

33 Goldenseal (Hydrastine canadensis; family Ranunculaceae) is a native North American plant 34 and has long been used in traditional medicine for its pharmacological activities such as 35 antibacterial [1], immunostimulant, antimicrobial [4] and anticancer properties [5]. Approximately 3644 alkaloids are described as main phytochemicals in the goldenseal plant [6]. The most abundant 37 alkaloids are berberine, hydrastine, and canadine (Figure 1), which have been recognized as the 38 active constituents for most pharmacological activities [7].

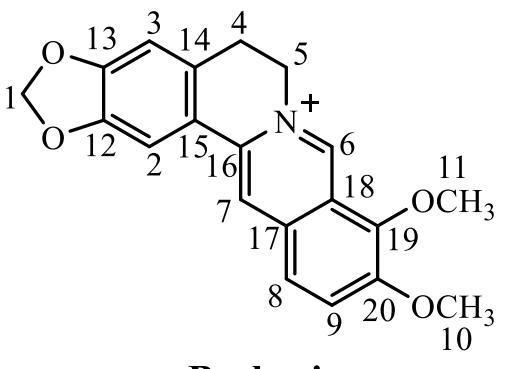

Berberine

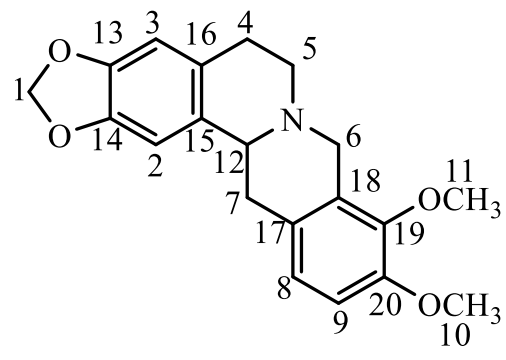

Canadine
39

40 Figure 1 Major alkaloids in goldenseal root extracts.

Today, this plant has emerged as one of the 20 most popular herbal supplements used worldwide. The global sales of goldenseal root as a dietary supplement in pharmaceutical outlets 
43 totaled US\$ 25 million in 2012 [10]. Due to the slow growing nature and the popularity of

44 goldenseal, several other plants containing berberine have been substituted for the plant. The 45 adulterants include Goldthread (Coptis japonica), Oregon grape root (Berberis aquifolium Pursh),

46 Barberry (Berberis spp.), and Yellow root (Xanthorhiza simplicissima). Most of these adulterants

47 contain berberine, but hydrastine and canadine are only known from goldenseal [11]. Therefore,

48 berberine, hydrastine and canadine are usually used as the markers and the analysis of these 3

49 alkaloids for the assessment and quality control of goldenseal products is an important step. Most

50 of the previously published studies have used LC-UV and TLC-MS methods for the identification

51 and quantitative analysis of berberine, canadine and hydrastine in goldenseal [13].

52 In recent years, quantitative Nuclear Magnetic Resonance (qNMR) has been widely used for 53 the analysis of different compounds in complex mixtures of plant extracts [16], drugs [17] and 54 foods [19]. The implementation of qNMR for identification and quantification of alkaloids in 55 herbal medicines, such as Rhizoma coptidis [20], Ephedra species [21], was also documented. 56 qNMR methods provide rapid, accurate, highly repeatable and reproducible quantification of 57 several components together with their unambiguous identification $[22,23]$. Therefore, qNMR 58 appears particularly adapted for the quality control of herbal samples [16].

59 In the quantitative NMR toolbox, $1 \mathrm{D}{ }^{1} \mathrm{H}$ is the most sensitive method to rapidly access 60 concentrations. In particular, $1 \mathrm{D}{ }^{1} \mathrm{H}$ has the advantage that multiple compounds can be quantified 61 in a single experiment relying on a unique reference signal, provided that experiments are carried 62 out under quantitative conditions [25]. However, due to the large number of substances having 63 different numerous signals and the broad associated range of concentrations, peak overlapping 64 issues can be highly challenging. Various approaches have made it possible to improve the 65 separation of overlapped resonances in qNMR, most of them relying on 2D NMR approaches [23], 

sometimes associated with fast acquisition methods [26]. While these 2D qNMR methods can reach

67 a good precision and trueness, they require standard addition or calibration approaches that rely on commercial standards. Alternative approaches have emerged to make 2D NMR intrinsically 69 quantitative, yielding quantitative results even when a commercial standard is not available [28,30].

70 Among these methods, we recently developed a faster quantitative approach named Q QUIPU 71 (Quick QUantItative Perfected and pUre shifted) HSQC, which combines several time-saving 72 strategies with quantitative pulse sequence elements. In this previous work, complex mixtures at 73 sub-millimolar concentrations could be quantified in 5 hours [31]. This highly dispersed and 74 sensitive method provides an appealing approach to quantify complex mixtures involving a broad 75 range of concentrations. However, when compounds are unstable, the experiment duration could 76 be prohibitive and one may still rely on 1D NMR, provided that the targeted analytes exhibit at 77 least one well-separated signal.

In this context, the main objective of this work is to explore the complementarity of qNMR methods, by combining the sensitivity and rapidity of $1 \mathrm{D}{ }^{1} \mathrm{H}$ with the dispersion power of $2 \mathrm{D} \mathrm{Q}-$ QUIPU HSQC to identify and quantify 3 natural products in Goldenseal extracts: berberine, canadine and hydrastine. In order to evaluate the accuracy of concentrations derived from the combined NMR strategy, a comparative study using UPLC-MS and UPLC-UV is also performed

83 to evaluate the performances of the combined NMR approach. 


\section{Materials and Methods}

90

\subsection{Plant Material}

92 Dried goldenseal roots used in this study were obtained from a Hamilton (ON, Canada) 93 goldenseal farm in May 2005. The plant material was authenticated by an ethnobotanist (Dr 94 Arnason at Ottawa University).

\subsection{Chemicals and reagents}

97 Certified reference materials berberine, hydrastine and canadine (CRM BERB-1, HYDR-1 and 98 CANA-1) with purities of $93.35 \%$; 99.32 and $99.56 \%$, respectively were obtained from National 99 Research Council Canada (Ottawa, Canada). Maleic acid, a reference material, with a purity of 100 99.35\%; was purchased from Fluka. Dimethyl sulfone with a purity of 98\%; was purchased from

101 Sigma-Aldrich. All organic solvents and formic acid were of HPLC grade and were purchased from 102 Fisher Scientific (ON, Canada). Ammonium formate was purchased from Sigma-Aldrich 103 (Oakville, ON, Canada). Ultra-pure water $(18.2 \mathrm{M} \Omega, \mathrm{cm})$ was produced in-house with a Thermo 104 Scientific GenPure filtration system. For NMR analysis: Deuterium dimethyl sulfoxide-d6 (99.9\% D) was provided by Cambride Isotope Laboratoires INC (Andover, MA, USA). NMR tubes (5 mm 106 o.d., length of 7", thin wall of $0.38 \mathrm{~mm}$ ) were from Wilmad-LabGlass Com. (Vineland, NJ, USA). 107

\subsection{Goldenseal extraction}

109 Dried goldenseal roots were milled to a powder (particle size less than $250 \mu \mathrm{m}$ ), thoroughly 110 homogenized using a rotary mixing drum and thereafter stored at $-80^{\circ} \mathrm{C}$. 
112 temperature for $20 \mathrm{~min}$ for $6 \times 200 \mathrm{ml}$ of a methanol-water-formic acid mixture (90-9.9-0.1; v/v/v)

113 as an extraction solvent. All extract solutions were pooled, and then filtered. The rest of the

114 supernatant liquid was evaporated down to dry under speed-vac and in the dry vacuum at room 115 temperature for one week. This procedure resulted in $10.6 \mathrm{~g}$ of dry crude extract of goldenseal root, 116 which was stored at $-20^{\circ} \mathrm{C}$ for further quantitative analysis by $1 \mathrm{D}$ and $2 \mathrm{D}$ NMR and also by UV 117 and MS analysis.

$119 \quad 2.4$ NMR analyses

120 2.4.1 NMR solvent and signal identification for major alkaloids in goldenseal

121 DMSO-d6 was chosen as an NMR solvent because all the three individual pure alkaloids and 122 the goldenseal extract were dissolved well in this solvent.

123 Approximately $10 \mathrm{mg}$ of berberine, canadine, and hydrastine were individually dissolved in 1 $124 \mathrm{ml}$ DMSO-d6 for recording 1D and 2D NMR analyses to assign the ${ }^{1} \mathrm{H}$ and ${ }^{13} \mathrm{C}$ NMR chemical 125 shifts of these 3 alkaloids in the NMR spectra of goldenseal extracts.

126 2D NMR spectra were recorded at $298 \mathrm{~K}$ on a Bruker Avance HD 700 spectrometer, at a 127 frequency of $700.13 \mathrm{MHz}$ with a warm TXI probe including z-axis gradients. DMSO-d6 was used 128 as the internal lock. Different 2D experiments like COSY, HSQC, and HMBC were performed to 129 identify major alkaloids of interest (see Table S1).

$132 \quad$ Five samples each containing 123.7; 117.7; 122.8; 123.4 and $115.6 \mathrm{mg}$, respectively, of dry 133 goldenseal extract were co-dissolved with $15 \mathrm{mM}$ of maleic acid as internal standard in $1 \mathrm{~mL}$ 134 DMSO- $\mathrm{d}_{6}$. The quantification of berberine in the goldenseal extract by ${ }^{1} \mathrm{H}$ qNMR was performed 
on a Bruker Avance III $600 \mathrm{MHz}$ at $20^{\circ} \mathrm{C}$. The samples were vortexed, sonicated, then centrifuged and $800 \mu \mathrm{L}$ of supernatant were loaded into a $5 \mathrm{~mm}$ NMR tube, sealed and used for quantitative

$137{ }^{1} \mathrm{H}$ qNMR measurements. Five goldenseal samples were analysed by ${ }^{1} \mathrm{H}-\mathrm{NMR}$ and each sample was measured in triplicate.

The ${ }^{1} \mathrm{H}$ qNMR acquisition was performed with a $90^{\circ}$ pulse, a sweep width of $30 \mathrm{ppm}(18,000$

$140 \mathrm{~Hz}$ ), 32 scans of $128 \mathrm{~K}$ data points preceded by 4 dummy scans, an acquisition time of $3.63 \mathrm{~s}$ and 141 a recovery delay of $35 \mathrm{~s}$, requiring $27 \mathrm{~min}$ per sample. In order to ensure the full relaxation of

$142{ }^{1} \mathrm{H}-\mathrm{NMR}$ resonance (including the internal standard) to obtain highly accurate ${ }^{1} \mathrm{H}$ qNMR spectra, 143 the longitudinal relaxation times (T1) were measured (prior to the quantitative ${ }^{1} \mathrm{H}$ qNMR 144 measurements) for a sample containing goldenseal extract and the maleic acid in DMSO-d6. From 145 this experiment, the longest relevant T1 was $4.45 \mathrm{~s}$, and the repetition delay was subsequently set 146 to $35 \mathrm{~s}$ (i.e., 7 times the longest T1) to ensure an accuracy of $0.1 \%$ with a $90^{\circ}$ pulse. Data processing 147 was performed using $64 \mathrm{~K}$ data points. The FIDs were Fourier transformed and apodized by an 148 exponential function leading to a line broadening of $0.3 \mathrm{~Hz}$ (LB). The resulting spectra were 149 manually phased and a manual polynomial baseline correction encompassing the desired integral 150 regions was carried out prior to integration. The reference frequency was set to the DMSO-d6 signal 151 at $2.50 \mathrm{ppm}$. All the spectra were integrated using TopSpin software (version 3.2, Bruker). 152

\subsubsection{Quantification of major alkaloids in goldenseal by 2D HSQC-NMR}

154 Three more samples were prepared with 100, 101 and $201 \mathrm{mg}$ of dried extract respectively 155 dissolved in $1 \mathrm{~mL}$ of DMSO-d6 including $2 \mathrm{mM}$ of $\mathrm{DMSO}_{2}$ as an internal reference.

156 For all 2D experiments, longitudinal relaxation ${ }^{1} \mathrm{H}$ times were measured and a recovery time 157 of $22 \mathrm{~s}$ was chosen for quantitative HSQCs. Here, 5 times the longest $\mathrm{T}_{1}$ time was considered as a 
compromise between a good accuracy and a reasonable experimental time for a precision of $1 \%$ 159 (see below). For the Q QUIPU HSQC, parameters were optimized according to reference [31]. The 2D HSQC maps were recorded in 2 days with $5452 \times 160$ data points. 2D matrices have been zero filled to $16384 \times 1024$ and apodized with a cosine in the two dimensions, prior to double Fourier transformation. 176 scans were recorded for each increment in the indirect dimension, to reach a minimum SNR of 50 ensuing a precision in the range of $1 \% .{ }^{1} \mathrm{~J}_{\mathrm{CH}}$ compensation was carried out by varying the INEPT delay throughout the successive scans (8 INEPTs in total: 6 with short delays $\Delta=1.47 \mathrm{~ms}$ and 2 with a longer delay $\Delta=2.96 \mathrm{~ms}$ ).

The pure shifting of the acquisition was performed with 10 chunks of $30 \mathrm{~ms}$ and $\mathrm{BIRD} / 180^{\circ}$ elements were used to ensure the absence $\mathrm{J}_{\mathrm{HH}}$ evolution during the acquisition time (300 ms). For each HSQC experiment, an echo-antiecho method and an adiabatic heteronuclear decoupling of

$17028 \mathrm{kHz}$ were used on the ${ }^{13} \mathrm{C}$ channel. Decoupling consisted of $1.5 \mathrm{~ms}$ smoothed CHIRP pulses 171 with a total sweep width of $60 \mathrm{kHz}$ and a P5M4 supercycle. Moreover, Q QUIPU is using 50\% 172 NUS meaning that $50 \%$ of the $t_{1}$ increments were actually acquired [32]. A Poisson gap sampling 173 schedule was used since it was shown to yield cleaner spectra after reconstruction. The 174 reconstruction of the $2 \mathrm{D}$ spectrum was performed using the compressed sensing algorithm 175 available within the Bruker software [33]. Compressed sensing generates less reconstruction 176 artefacts than recursive multidimensional decomposition (MDD) for 2D spectra [34]. A semi177 gaussian/linear function was been used as Variable repetition time (VRT) function [31]. A two-fold 178 aliasing was implemented leading to a $65 \mathrm{ppm}$ apparent spectral width centered on $52.5 \mathrm{ppm}$ along 179 the ${ }^{13} \mathrm{C}$ dimension. Before Fourier tranform, 2D maps were weighted with cosine functions over 180 the two dimensions. The resulting spectra were manually phased, an automatic polynomial baseline 
correction and referenced to the residual DMSO-d6 signal at $2.50 \mathrm{ppm}$. The peak volumes were 182 measured using the $2 \mathrm{D}$ integration tool in Topspin 3.2. The integration region limits were 183 systematically optimized for each peak, on the metabolite model sample with the following criteria 184 (i) integration regions centred on the peak of interest and (ii) integration regions size chosen as 185 large as possible to integrate the whole peak but small enough to avoid overlap with neighboring peaks and integration regions.

Three goldenseal samples were analysed by 2D NMR and each sample was measured in 188 triplicate.

189 In both $1 \mathrm{D}-{ }^{1} \mathrm{H}[35]$ and $2 \mathrm{D}$ Q QUIPU-HSQC experiments, the area of a signal appearing on a 190 NMR spectrum is directly proportional to the concentration as well as the number of resonant nuclei 191 corresponding to the signal.

\subsection{UPLC-UV and UPLC-MS-MS analysis}

194 Stock solutions of berberine, hydrastine and canadine (standard compounds) at a concentration 195 of $1.0 \mathrm{mg} / \mathrm{mL}$ in methanol were individually prepared and stored at $-20^{\circ} \mathrm{C}$ for no longer than 1 196 month. Using these individual alkaloid stock solutions, a mixed standard solution at an appropriate 197 concentration was prepared. The external standard calibration curve was prepared at seven different 198 concentration levels by the serial dilution with $\mathrm{MeOH}$ of the mixed standard solution. Finally, the 199 range of the external calibration curve was from 0.1 to $145 \mu \mathrm{g} / \mathrm{mL}$ for UPLC-UV and from 0.002 200 to $1.45 \mu \mathrm{g} / \mathrm{mL}$, for UPLC-MS-MS analysis.

201 The stock solution of goldenseal extract was prepared in $\mathrm{MeOH}$ at concentration of $1.20 \mathrm{mg} / \mathrm{ml}$ 202 and then diluted 20 and 100 times, respectively, for UPLC-UV and UPLC-MS analysis. 7 samples 203 of goldenseal extract were prepared. The volume of $2 \mu \mathrm{l}$ of working calibration solution and 
204 goldenseal sample was injected in triplicate into the UV and MS system. All the solutions of 205 samples and standards were gravimetrically prepared.

206 Analyses were performed with an UPLC (Infinity 1290, Agilent Technologies) system 207 equipped with an Agilent diode array detector (DAD) and/or an ESI-triple quadrupole mass 208 spectrometer (Quantiva TSQ, Thermo Scientific). The samples were separated with an Acquity 209 UPLC Beh C18 column $100 \mathrm{~mm}$ x $2.1 \mathrm{~mm}, 1.7 \mu \mathrm{m}$ at $40^{\circ} \mathrm{C}$. The mobile phase consisted of A, 210 water containing $15 \mathrm{mM}$ ammonium formate adjusted to $\mathrm{pH} 3.8$ with formic acid, and $\mathrm{B}$, mixture 211 of $15 \mathrm{mM} \mathrm{NH}_{4}$ Formate, $\mathrm{pH} 3.8$ and acetonitrile; 10:90 (v/v). The flow rate was maintained at 0.4 $212 \mathrm{~mL} / \mathrm{ml}$ and the following gradient program was used: $19-30 \% \mathrm{~B}$ from 0 to $7 \mathrm{~min} ; 30-95 \% \mathrm{~B}$ from 2137 to $8 \mathrm{~min}$; hold at $95 \% \mathrm{~B}$ from 8 to $10 \mathrm{~min}$. The total run time for the analysis was $10 \mathrm{~min}$. The 214 standards and goldenseal sample were kept at $10^{\circ} \mathrm{C}$ during analysis. The $2 \mu \mathrm{L}$ of working 215 calibration solution and goldenseal sample were injected in triplicate into the UV and MS system.

216 The UV chromatograms were recorded at 290, 294 and $344 \mathrm{~nm}$ for canadine, hydrastine and 217 berberine, respectively (Table S2). The DAD spectra from 190 to $600 \mathrm{~nm}$ were stored for all peaks. 218 The concentrations of berberine, hydrastine and canadine in goldenseal sample were determined 219 by using the UV detected peak area under the curve to the established calibration curves made from 220 the standard compounds. Data processing was performed using the LAB CDS ChemStation 221 software for UV analysis.

222 The MS detection was carried out in positive ion mode, using SRM (selected reaction 223 monitoring) mode by monitoring MS/MS transitions $m / z$ 336.1/292.0 for berberine and 384.1/190.1

224 for hydrastine and 340.1/176.1 for canadine (Table S2). Experimental conditions for ESI ionization 225 and mass spectrometry detection are summarized in Table 1. Quantitative MS analysis was 226 performed in the SRM mode. The peak area of the SRM ion of berberine, canadine and $\beta$ - 
227 hydrastine was used to calculate the concentration based on the standard calibration curve. Data 228 processing was performed using the Xcalibur software for MS analysis.

229 Seven goldenseal samples were analysed by UPLC-UV and UPLC-MS-MS and each sample 230 was measured in triplicate. In addition, the UPLC-MS-MS and UPLC-UV methods were validated 231 in terms of linearity, precision, stability, repeatability and recovery (data not shown). Details on the 232 validation of quantitative UPLC-MS-MS and UPLC-UV methods of alkaloids in goldenseal will 233 be published elsewhere.

\subsection{Extract optimization}

236 Various solvent systems were evaluated to obtain the maximum recoveries of 3 major alkaloids 237 (berberine, canadine and hydrastine) in goldenseal root powder, including 100\%; 90\%; 80\% 238 methanol (or acetonitrile $\mathrm{ACN}$ ); $30 \%$ methanol (or acetonitrile) $+0.1 \%$ phosphoric acid; $90 \%$ $239 \mathrm{MeOH}$ (or acetonitrile) $+0.1 \%$ Formic acid; $90 \% \mathrm{MeOH}$ (or acetonitrile) $+0.1 \%$ acetic acid. The 240 recoveries determined by LC-MS of the major alkaloids were found to be higher (>97\%) with 90 $241 \%$ methanol/water/0.1\% Formic acid (v/v/v) than with other solvents. The sonication time was also 242 optimized. 20 and 30 min were tested and no significant increase was observed with the extended 243 treatment times. 20 min was selected as optimum extraction period. There was no analyte observed 244 after the $4^{\text {th }}$ extraction by LC-UV detector and $6^{\text {th }}$ by LC-MS detector. The sixth extraction of 245 samples was considered to be exhaustive.

\section{Results and Discussion}

248 As mentioned earlier, the choice of the most appropriate quantitative NMR tool depends on 249 criteria such as 1D peak overlap, concentration, and stability in time. Here, we evaluate both 1D 
$250 \quad{ }^{1} \mathrm{H}$ NMR and 2D Q QUIPU for the quantification of the 3 major alkaloids in goldenseal extract 251 (Figure 1).

2523.1 Potential of $1 D^{1} \mathrm{H}$ NMR for quantification

253 The easiest way to determine concentrations is the $1 \mathrm{D}{ }^{1} \mathrm{H}$ experiment that is also one of the 254 most sensitive experiments. The representative ${ }^{1} \mathrm{H}-\mathrm{NMR}$ spectra of individual alkaloids and of the 255 goldenseal extract are shown in Figure 2. The assignment of NMR signals for each alkaloid was 256 based on experiments performed on standard solutions (see Table S1).

257 Signals of the major alkaloids in the extracts are indicated in Figure 2. Accurate quantitative $258{ }^{1} \mathrm{H}$ NMR, is possible when the analyte of interest shows at least one well-separated signal. In the 259 case of goldenseal extract, berberine has two characteristic signals at 9.89 and 8.94 ppm 260 (corresponding to $\mathrm{H}_{6}$ and $\mathrm{H}_{7}$ respectively, see Figure 1) that are isolated and easily identified since 261 they do not overlap with other signals in the ${ }^{1} \mathrm{H}$ spectrum of goldenseal extract. On the contrary, 262 all the peaks of hydrastine were at least partially overlapped by those of minor compounds in the $263{ }^{1} \mathrm{H}$ spectrum, making accurate quantification very difficult by $1 \mathrm{D}$ NMR. It is even worse for 264 canadine, which could not be detected due to its low concentration and to the large number of 265 compounds giving rise to overlapping peaks in corresponding spectral region (Figure 2a).

266 


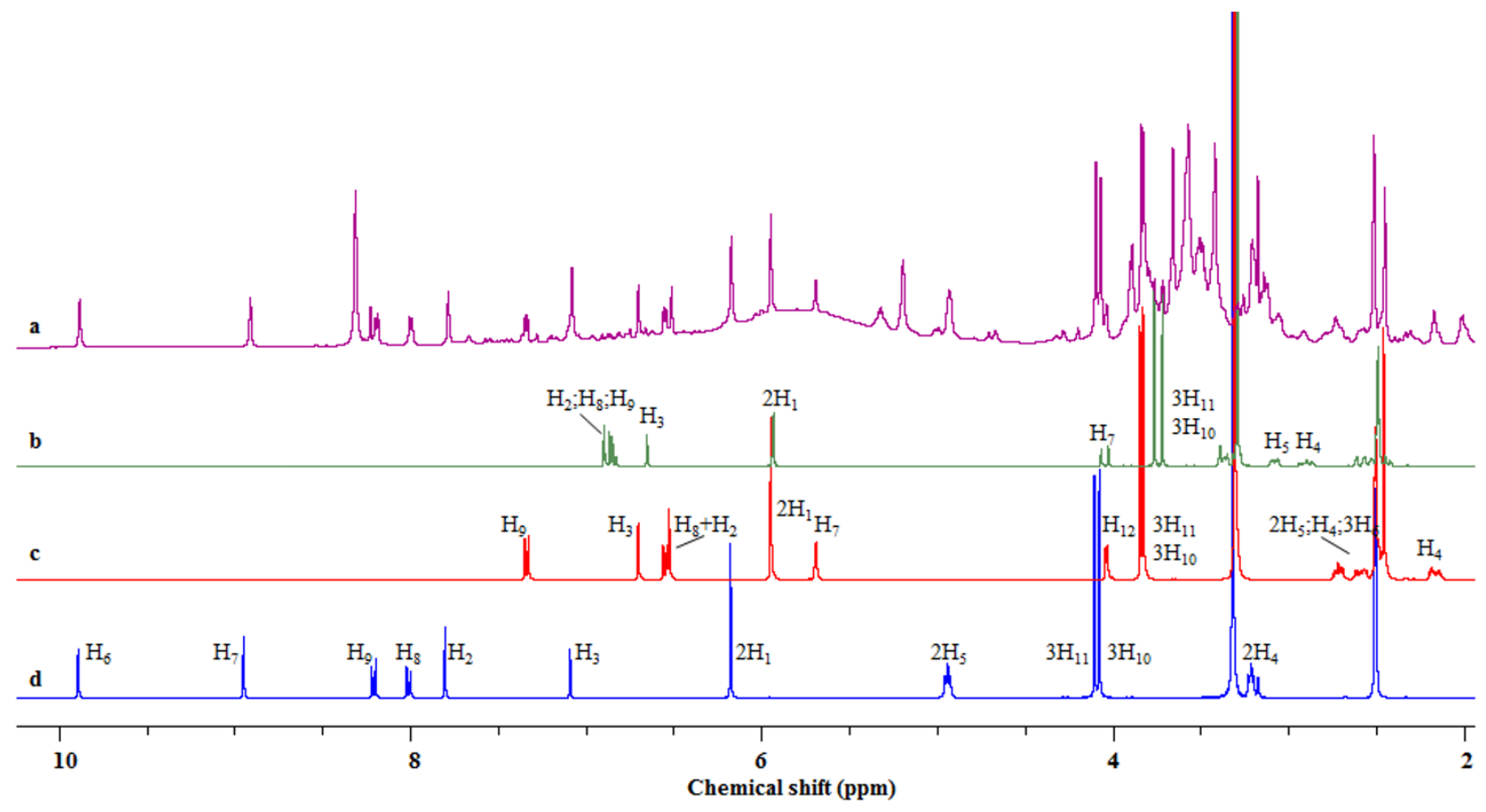

272 Figure $2{ }^{1} \mathrm{H}$ NMR spectra of goldenseal extract (a), canadine (b) $\beta$-hydrastine (c) and berberine

273 (d). More details on the assignment can be found in Table S1.

274 There is no need to use calibration curves in ${ }^{1} \mathrm{H}$ qNMR analysis, since the signal intensity is 275 directly proportional to the number of resonant nuclei contributing to each signal [22]. The 276 concentration of the compounds can be calculated by the relative ratio of the peak integral of each 277 compound to the integral of the peak of a known amount of internal standard (IS), accounting for 278 the number of protons in each integral. In this study, we chose maleic acid as an IS for the following 279 reasons: it is not present in goldenseal, it is a reference material with a high purity, it is a non280 hygroscopic and easily weighable solid with a good solubility and stability in DMSO-d6, and its 281 unique signal does not overlap with other signals (see Figure S1). As mentioned above, the two proton signals of berberine (proton $\mathrm{H}_{6}$ and $\mathrm{H}_{7}$ ) were selected to quantify berberine in the goldenseal extract by ${ }^{1} \mathrm{H}$ qNMR. As shown in Table S3, the concentrations of berberine (obtained during the first two hours, before the degradation) in mg per gram of goldenseal extract by ${ }^{1} \mathrm{H}$ qNMR were in 
285 the range $105.37 \mathrm{mg} / \mathrm{g}$ (SD:1.2). The results obtained by ${ }^{1} \mathrm{H}$ NMR (see Table S3) are consistent 286 from different measurements based on triplicate and from sample to sample.

\subsection{DQ QUIPU capabilities for quantifying goldenseal alkaloids}

289 Due to the limitations of ${ }^{1} \mathrm{H}-\mathrm{qNMR}, 2 \mathrm{D}$ Q QUIPU HSQC experiments were carried out in order 290 to quantify the two other major alkaloids in the goldenseal root extract, hydrastine and canadine 291 (see Figures S2). As previously shown on breast cancer cell extracts, this ${ }^{1} \mathrm{H}-{ }^{13} \mathrm{C} 2 \mathrm{D}$ experiment 292 provides a better sensitivity than standard HSQC: +26 to $92 \%$ as compared to the non-pure-shift 293 version (see Figure S3) [28]. The concentrations of $\beta$-hydrastine, and canadine were accurately 294 obtained from 2D Q QUIPU maps (see Figure 3) by using $\mathrm{DMSO}_{2}$ as an internal reference.

295 The different quantities of the main alkaloids were obtained by averaging all cross peak 296 volumes for each alkaloid of interest (some of them can be seen in Figure 3) deriving from 2D Q 297 QUIPU maps (see Figure S2). In order to evaluate the associated repeatability, 3 spectra were 298 recorded for each sample, leading to a good precision between 1 and 7\%. Moreover, 3 different 299 samples were prepared to evaluate the reproducibility (ranging from 0.3 to $1.2 \%$ ) (see Figure S4 300 and Table S4). 

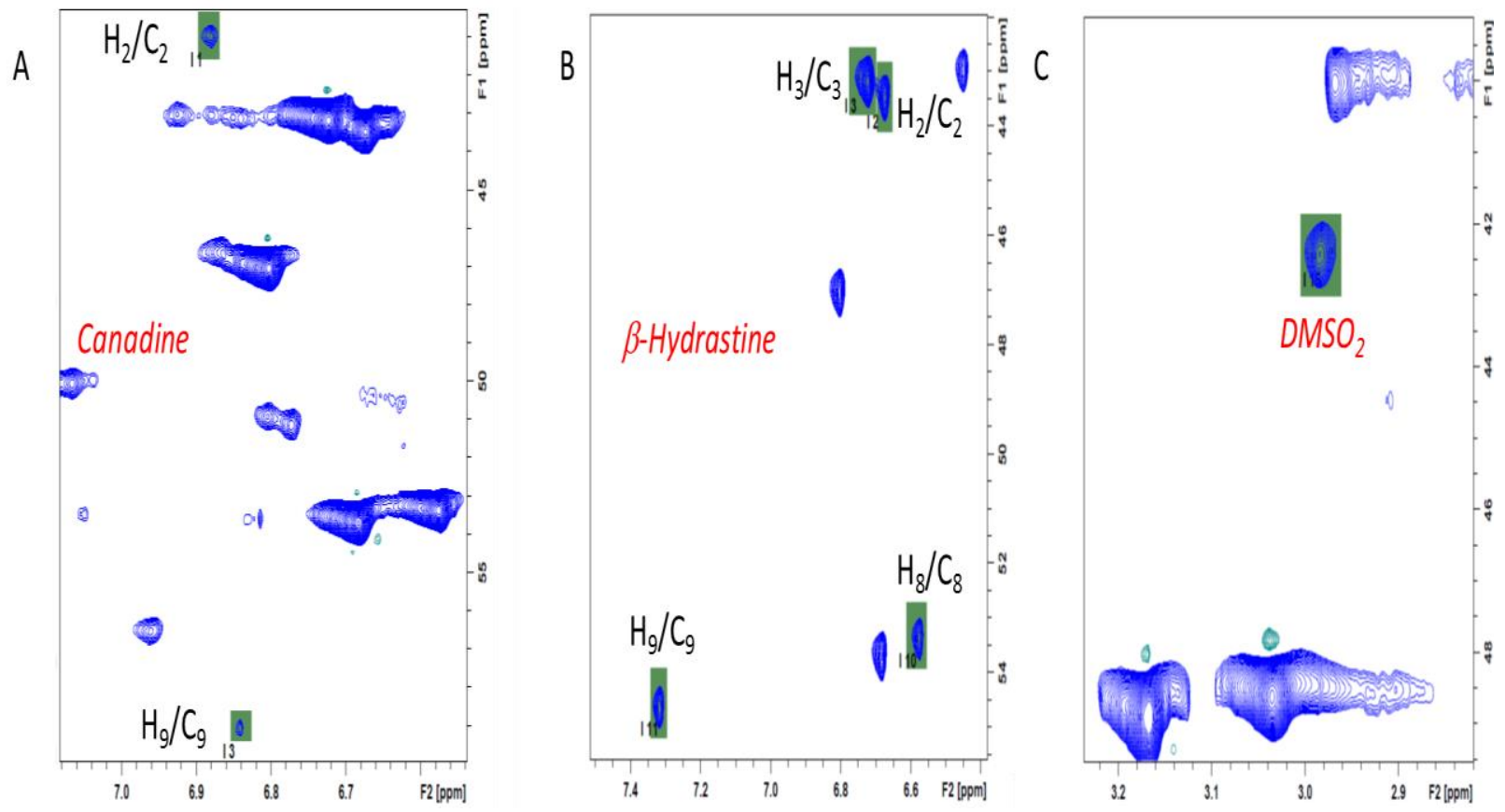

302

Figure 3 Parts of 2D Q QUIPU map showing canadine (A), $\beta$-hydrastine (B), and $\mathrm{DMSO}_{2}(\mathrm{C})$ signals with the corresponding integration regions (green squares). $\mathrm{DMSO}_{2}$ was used as an internal 304 reference.

305

\subsection{Complementarity of $1 D^{1} \mathrm{H}$ and $2 D Q Q U I P U$}

307 Berberine has shown a tendency to degrade within the first two hours after the sample 308 preparation. Under such conditions, $1 \mathrm{D}{ }^{1} \mathrm{H}$ is the only experiment able to rapidly detect signals of 309 the berberine (see Figure 2) with the best accuracy and repeatability (see Table S3) in only 3 min. 310 In this context the Q QUIPU is not competitive to determine the concentration of berberine. On the 311 contrary, probing the quantity of hydrastine and canadine with $1 \mathrm{D}{ }^{1} \mathrm{H}$ by integration is difficult due 312 to overlaps (see Figure 2). In the case of such overlap, spectral deconvolution of 1D spectra could 313 be suggested as an alternative [36,38]. Thus, a global spectral deconvolution (GSD) using the tool 314 implemented in Mnova 12 was performed. However, the accuracy of the resulting concentrations 315 for hydrastine and canadine is disappointing - between 14 and $55 \%$ as compared to 2D Q QUIPU 
316 (Figure S5B and Table S5 in ESI) - and 2D NMR appears in much better agreement with the results

317 obtained by MS. This shows the limits of the deconvolution approach and highlights the superiority 318 of the quantitative 2D NMR approach in the case of strong overlap.

319 Although Q QUIPU showed a great ability to access the lower concentration of canadine, 2 320 days of experiment were required to reach a SNR close to 50 ensuring a precision of $1 \%$. By using 321 a cryogenically cooled probe which was not available at the time of the study, it could be possible 322 to reduce the experimental time by a factor from 9 to 16 , leading to a total time from 3 to $5 \mathrm{~h}$ for 323 the 2D Q QUIPU with the same level of accuracy.

324 In order to evaluate the quantitative performance of the combined NMR approach, other MS325 based analytical techniques were used for comparison.

\subsection{UPLC-UV and UPLC-MS-MS analysis}

328 In parallel to the quantification of 3 major alkaloids (berberine, canadine and hydrastine) in 329 goldenseal extract by 1D and 2D-qNMR (as above), we developed the quantification of these three 330 alkaloids by other independent methods such as UPLC-UV and UPLC-MS-MS. The quantification 331 of 3 major alkaloids in goldenseal material has been published in previous studies [13, 14], but not 332 for the same goldenseal material which was used in our study.

333 The presence of berberine, $\beta$-hydrastine and canadine in goldenseal extract was already 334 confirmed in our previous publication $[6,40]$ by comparison of their retention time, UV wavelength 335 absorbance, MS precursor ion and MS-MS fragmentation ions to those of standard compound.

336 Here, berberine, $\beta$-hydrastine and canadine in goldenseal extract were quantified using the 337 corresponding calibration curves of each compound for UPLC-UV and UPLC-MS-MS method 338 leading to precision from 0 to $1 \%$ (see Table 1 ). 


\subsection{Comparison of the combined NMR quantification with MS-based methods}

341 For the most concentrated alkaloids (berberine and hydrastine), concentrations derived from

342 NMR are in very good agreement with those obtained by UPLC-UV/MS with trueness in the range

343 of 1 to $3 \%$ (Table 1 and Figure 4). Despite the longer acquisition times of the 2D Q QUIPU

344 technique, it was possible to quantify the lowest concentrated canadine in the range of $6 \mathrm{mg} / \mathrm{g}$ in 2

345 days with a good accuracy: 1-3\% regarding UPLC-MS, and an acceptable one in the range of 0.3

346 to $9.6 \%$ as compared to UPLC-UV (see Table 1 from the $6^{\text {th }}$ to the $7^{\text {th }}$ columns). The quantities of

347 canadine obtained by UPLC-UV are higher than those obtained by UPLC-MS and 2D-NMR (see

348 Table 1 column 2 versus 3 and 5 columns), which may be explained by the peak overlap between

349 canadine and other compound resulting from the LC-UV method. Using this method, the quantities

350 of canadine was measured based on its UV detected peak area at $290 \mathrm{~nm}$ (Table S2) under the

351 standards calibration curve. This method often lacks highly compound specific information,

352 especially for compound with low concentration such as canadine in the complex goldenseal

353 extract, because other compound having a similar retention time on the UV chromatography are

354 also absorbed at around $290 \mathrm{~nm}$. In contrast, the quantities of canadine obtained by the UPLC-MS

355 method using the SRM mode was determined based on both MS precursor ion at $m / z, 341.1$ and

356 MS-MS fragmentation ion at $m / z, 176.1$ (Table S2) and also based on the retention time determined

357 from the calibrations with standard compounds. Therefore, the quantitative conditions of canadine

358 in complex goldenseal extract by UPLC-MS are more selective than those of UPLC-UV, probably

359 leading some discrepancy for canadine amount obtained by UPLC-UV and UPLC-MS. The indices

360 above could be partly explained by reminding that the proposed quantitative NMR approach is

361 more accurate than indirect methods such as UV and MS, because the higher number of steps 
362 required in indirect methods can increase time consumption and introduce sources of bias due to a 363 lower accuracy.

364 In a general point of view, the quantification by NMR can be made directly on the matrices in 365 the absence of further purifications and does not rely on external calibration because the NMR 366 signal is molar equivalent. With the addition of a known concentration of IS to the matrices 367 (goldenseal extract in this case), the concentration of compound in the matrices can easily be 368 calculated. This is a great advantage when it is difficult to obtain pure standard compounds. An 369 advantage of the qNMR method over UPLC-UV and MS methods is that it requires much less 370 sample preparation time and no calibration curves for quantification are necessary.

\begin{tabular}{|c|c|c|c|c|c|c|}
\hline Compound & 1D ${ }^{1}$ H-NMR & 2D Q-QUIPU & UPLC-UV & $\begin{array}{c}\text { UPLC-MS- } \\
\text { MS }\end{array}$ & $\begin{array}{c}\text { Accuracy } \\
(\%) \\
\text { NMR/UV }\end{array}$ & $\begin{array}{c}\text { Accuracy } \\
(\%)\end{array}$ \\
NMR/MS \\
Berberine & $105.37(1.26)$ & NA & $105.08(0.96)$ & $\begin{array}{c}106.24 \\
(0.76)\end{array}$ & 0.27 & 0.82 \\
Hydrastine & NQ & $67.01(2.10)$ & $65.93(0.81)$ & $65.20(1.32)$ & 9.60 & 1.86 \\
Canadine & NQ & $5.91(0.29)$ & $6.48(0.10)$ & $6.02(0.27)$ & & \\
\hline
\end{tabular}

372

373 Table 1 Comparison of the alkaloid concentrations in GDS extract $(\mathrm{mg} / \mathrm{g} \pm \mathrm{SD})$ determined 374 independently by NMR (n=3), UV and by MS $(n=5)$.

375 NQ: not quantified due to the peak overlaps issue in $1 \mathrm{D}{ }^{1} \mathrm{H}$ NMR

376 NA: not applicable due to the long acquisition of 2D Q QUIPU NMR 


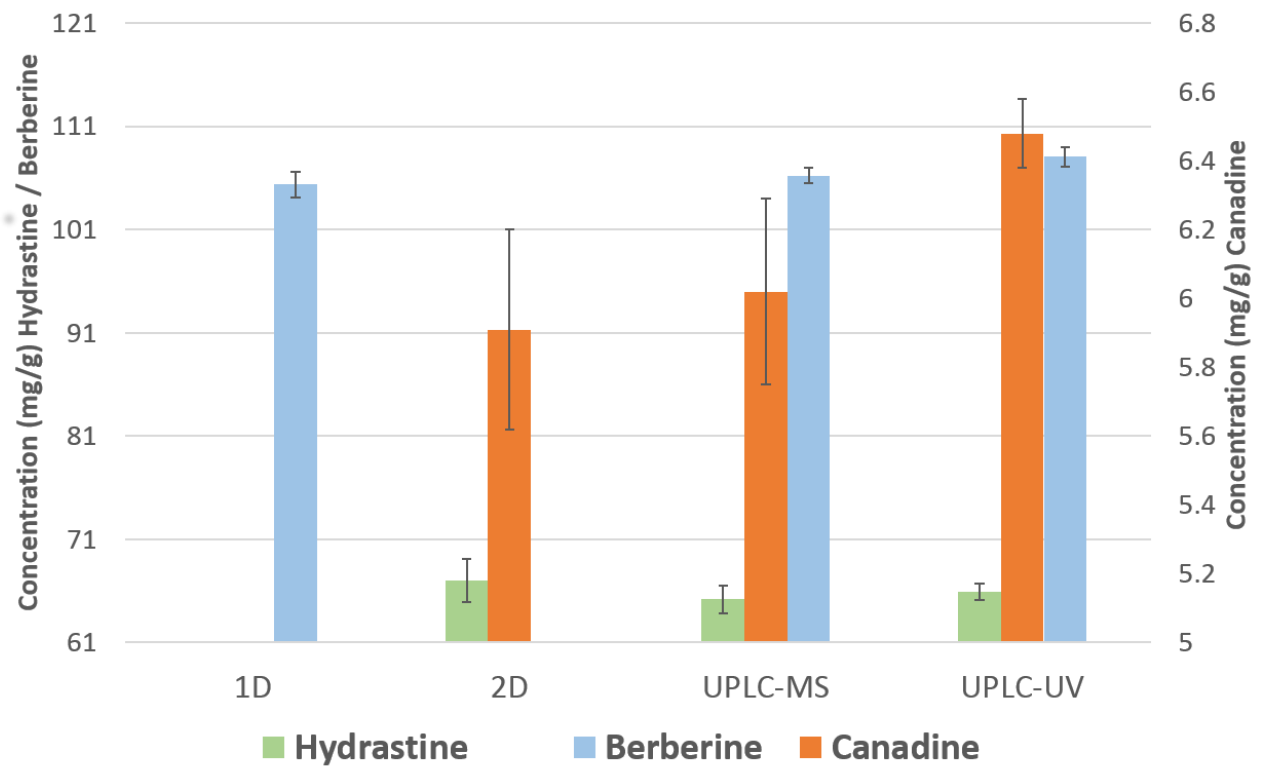

379 Figure 4 Concentration of berberine, canadine and $\beta$-hydrastine derived from 3 successive 2D Q 380 QUIPU experiments recorded over 3 samples in comparison with results obtained from triplicate 381 UPLC-UV and UPLC-MS over 5 samples. 4 Conclusions

2D advanced techniques presented in combination with $1 \mathrm{D}{ }^{1} \mathrm{H}$ NMR have shown their potential 384 for quantifying alkaloids of interest from goldenseal. Despite a longer time requiring 2 days of 385 measurement with a room temperature probe it was possible to access the quantity of the low 386 concentrated canadine at $6 \mathrm{mg} / \mathrm{g}$ of extract. Of course under this condition, it was difficult to 387 determine the concentration of berberine due to its degradability within the first hour after sample 388 preparation. The precision rate was very good in the range of 1 to $7 \%$. Overall, the concentration 389 of berberine, hydrastine and canadine obtained by ${ }^{1} \mathrm{H}$ and/or $2 \mathrm{D}$ qNMR measurement are in 390 acceptable to good agreements with those obtained by MS and UV measurement. The trueness was 391 compared to the data obtained with the coupled UPLC-MS and UPLC-UV leading to variability of 3921 to $10 \%$. Moreover, the ${ }^{1} \mathrm{H} 1 \mathrm{D}$ remains the direct method of choice for the quantification of natural 
393 products having moderate overlaps and possible unstable compound in the time. The combination

394 of different NMR techniques is the key to overcome limitations for investigating complex natural 395 mixtures embedding unstable and low concentrated molecules. In this field, 2D ultrafast techniques $396[26,27]$ combined with hyperpolarization methods [42,43] could be useful for enhancing the 397 resolution in seconds to minutes acquisition times with sensitivity enhancement from two to three 398 order of magnitude.

399 Thus, combined NMR approaches can be used and expanded to quantify other compounds of 400 interest in complex mixtures with a better sensitivity, and resolution, high precision, good accuracy, 401 repeatability and reproducibility and in a less time consuming manner in different areas such as 402 agricultural, food and/or pharmaceutical products.

403

404 Acknowledgments:

405 The authors from Nantes acknowledge the CORSAIRE Metabolomics platform (Biogenouest 406 Network). JF acknowledges CNRS and the University of Nantes for funds. He warmly thanks his 407 partner Sandrine Bouchet for an unfailing assistance. PML would like to thank Donald Leek (NRC 408 in Ottawa) for technical assistance in ${ }^{1} \mathrm{H}-\mathrm{NMR}$ experiments.

\section{$410 \quad$ References}

411 [1] F. Scazoccio, M.F. Cometa, L. Tomassini, M. Palmery, Anterbacterial activity of Hydrastis 412 canadensis extract and its major isolated alkaloids. Planta Med. 67 (2001) 561-564.

413 [2] K.A. Ettefagh, J.T. Burns, H.A. Junio, G.W. Kaatz, N.B. Cech, Goldenseal (Hydrastis 414 canadensis L.) extracts synergistically enhance the antibacterial activity of berberine vial efflux 415 pump inhibition. Planta Med. 77 (2011) 835-840. 
[3] R. Jalees, J.M. Dillow, S.M. Carter, J. Chou, B. Le, A.S. Maisel, Increased production of antigenspecific immunoglobulins $\mathrm{G}$ and $\mathrm{M}$ following in vivo treatment with the medicinal plants Echinacea angustifolia Hydrastis canadensis. Immunol. Lett. 68 (1999) 391-395.

[4] B.Y. Hwang, S.K. Roberts, L.R. Chadwick, C.D. Wu, A.D. Kinghorn, Antimicrobial constituents from goldenseal (the Rhizomes of Hydrastis canadensis) against selected oral pathogens. Planta Med. 69 (2003) 623-627.

[5] S.R. Karmakar, S.J. Biswas, A.R. Khuda-Bukhsh, Anti-carcinogenic Potentials of a Plant Extract (Hydrastis canadensis): I Evidence from in vivo studies in mice (Musmusculus). Asian Pacific Journal of Cancer Prevention 11 (2010) 545-551.

[6] P.M. Le, M. McCooeye, A. Windust, Application of UPLC-QTOF-MS in MSE mode for the rapid and precise identification of alkaloids in goldenseal (Hydrastis canadensis). Anal. Bional. Chem. 406 (2014) 1739-1749.

[7] E.J. Gentry, H.B. Jampani, A. Kesharvarz-Shokri, M.D. Morton, D. VanderVelde, H. Telikepalli, L.A. Mitscher, Antitubercular natural products: Berberine from the roots of commercial Hydrastis canadensis powder. Isolation of inactive 8-oxotetrahydrothalifendine, canadine, b-hydrastine, and two new quinic acid esters,hydcandinic acid esters-1 and2. J. Nat. Prod. 61 (1998) 1187-1193.

[8] P. Bolle, M.F. Cometa, M. Palmery, P. Tucci, Response of rabbit detrusor muscle to total extract and major alkaloids of Hydrastis canadensis. Phytother. Res. 12 (1998) S86-S88.

[9] H. Abdel-Haq, M.F. Cometa, M. Palmery, M.G. Leone, B. Silvestrini, L. Saso, relaxant effects of Hydrastis canadensis L. and its major alkaloids on guinea pig isolated trachea. Pharmacol. Toxicol. 87 (2000) 218-222.

[10] I.A.F.R.O. Cancer, Goldenseal. In Some Drugs and Herbal Products, World Health Organization: France, 2016; Vol. 108, pp 73-86.

[11] C. Cavaliere, P. Rea, M.E. Lynch, M. Blumenthal, Herbal supplement sales experience - slight rise in 2008. HerbalGram (2009) 58-61.

[12] R. Upton, Goldenseal Root (Hydrastis canadensis): Standard of Analysis, Quality Control, and Therapeutics. (2001) 10-18.

[13] B. Avula, Y.H. Wang, I.A. Khan, Quantitative Determination of Alkaloids from Roots of Hydrastis canadensis L. and Dietary Supplements Using Ultra-Performance Liquid Chromatography with UV Detection. Journal of AOAC Int. 95 (2012) 1398-1405. 
[14] P.N. Brown, M.C. Roman, Determination of hydrastine and berberine in goldenseal raw materials, extracts, and dietary supplements by high performance liquid chromatography with UV: Collaborative Study. J. AOAC Int. 91 (2008) 694-701.

[15] G.J. Van Berkel, B.A. Tomkins, V. Kerterz, Thin-layer chromatography/desorption electrospray ionization mass spectrometry: investigation of goldenseal alkaloids. Anal. Chem. 79 (2007) 2778-2789.

[16] S.K. Chauthe, R.J. Sharma, F. Aqil, R.C. Gupta, I.P. Singh, Quantitative NMR: An Applicable Method for Quantitative Analysis of Medicinal Plant Extracts and Herbal Products. Phytochem. Anal. 23 (2012) 689-696.

[17] A.A. Salem, H.A. Mossa, Method validation and determinations of levofloxacin, metronidazole and sulfamethoxazole in an aqueous pharmaceutical, urine and blood plasma samples using quantitative nuclear magnetic resonance spectrometry. Talanta 88 (2012) 104114.

[18] I. Berregi, G.D. Campo, R. Caracena, J.I. Miranda, Quantitative determination of formic acid in apple juices by $1 \mathrm{H}$ NMR spectrometry. Talanta 72 (2007) 1049-1053.

[19] B. Gouilleux, J. Marchand, B. Charrier, G.S. Remaud, P. Giraudeau, High-throughput authentication of edible oils with benchtop Ultrafast 2D NMR. Food Chemistry 244 (2018) 153-158.

[20] G. Fan, M.Y. Zhang, X.D. Zhou, X.R. Lai, Q.H. Yue, C. Tang, W.Z. Luo, Y. Zhang, Quality evaluation and species differentiation of Rhizoma coptidis by using proton nuclear magnetic resonance spectroscopy. Analytica Chimica Acta 747 (2012) 76-83.

[21] H.K. Kim, Y.H. Choi, W.T. Chang, R. Verpoorte, Quantitative Analysis of ephedrine analogues from Ephedra species using 1H- NMR. Chem. Pharm. Bull. 12 (2003) 1382-1385.

[22] G.F. Pauli, B.U. Jaki, D.C. Lankin, Quantitative 1H NMR: Development and Potential of a Method for Natural Products Analysis. J. Nat. Prod. 68 (2005) 133-149.

[23] P. Giraudeau, Quantitative 2D liquid-state NMR. Magn. Reson. Chem 52 (2014) 259-272.

[24] J. Zhao, M. Wang, B. Avula, L. Zhong, Z. Song, Q. Xu, S. Li, I.A. Khan, Effect of Processing on the Traditional Chinese Herbal Medicine Flos Lonicerae: An NMR-based Chemometric Approach. Planta Med. 81 (2015) 754-764.

[25] P. Giraudeau, I. Tea, G.S. Remaud, S. Akoka, Reference and normalization methods: Essential tools for the intercomparison of NMR spectra. J. Pharm. Biomed. Anal. 93 (2014) 3-16. 
[26] T. Jézéquel, C. Deborde, M. Maucourt, V. Zhendre, A. Moing, P. Giraudeau, Absolute quantification of metabolites in tomato fruit extracts by fast 2D NMR. Metabolomics 11 (2015) 1231-1242.

[27] L. Rouger, B. Gouilleux, M. Pourchet-Gellez, J.N. Dumez, P. Giraudeau, Ultrafast doublequantum NMR spectroscopy with optimized sensitivity for the analysis of mixtures. Analyst 141 (2016) 1686-1692.

[28] C. Mauve, S. Khlifi, F. Gilard, G. Mouille, J. Farjon, Sensitive, highly resolved, and quantitative (1)H-(13)C NMR data in one go for tracking metabolites in vegetal extracts. Chem. Commun. 52 (2016) 6142-6145.

[29] S. Khlifi, C. Mauve, N. Glab, F. Gilard, M. Hodges, G. Mouilled, J. Farjon, An easier analysis of complex mixtures with highly resolved and sensitivity enhanced 2D quantitative NMR: application to tracking sugar phosphates in plants. Analytical Methods 9 (2017) 2328-2333.

[30] K. Hu, W.M. Westler, J.L. Markley, Simultaneous Quantification and Identification of Individual Chemicals in Metabolite Mixtures by Two-Dimensional Extrapolated Time-Zero 1H-13C HSQC (HSQC0). J. Am. Chem. Soc. 133 (2011) 1662-1665.

[31] J. Farjon, C. Milande, E. Martineau, S. Akoka, P. Giraudeau, The FAQUIRE Approach: FAst, QUantitative, hIghly Resolved and sEnsitivity Enhanced 1H, 13C Data. Anal. Chem. 90 (2018) 1845-1851.

[32] B. Worley, R. Powers, Deterministic multidimensional nonuniform gap sampling. J. Mag. Reson. 261 (2015) 19-26.

[33] A. Stern, J. Hoch, A new approach to compressed sensing for NMR. Magn. Reson. Chem. 53 (2015) 908-912.

[34] V.Y. Orekhov, V.A. Jaravine, Analysis of non-uniformly sampled spectra with multidimensional decomposition. Prog. Nucl. Magn. Reson. Spectrosc. 59 (2011) 271-292.

[35] T. Rundlöfa, M. Mathiassona, S. Bekiroglub, B. Hakkarainena, T. Bowden, T. Arvidssona, Survey and qualification of internal standards for quantification by $1 \mathrm{H}$ NMR spectroscopy. J. Pharm. Biomed. Anal. 52 (2010) 645-651.

[36] R.S. Phansalkar, C. Simmler, J. Bisson, S.N. Chen, D.C. Lankin, J.B. McAlpine, M. Niemitz, G.F. Pauli, Evolution of Quantitative Measures in NMR: Quantum Mechanical qHNMR Advances Chemical Standardization of a Red Clover (Trifolium pratense) Extract. J. Nat Prod. 80 (2017) 634-647. 
[37] J.G. Napolitano, T. Gödecke, D.C. Lankin, B.U. Jaki, J.B. McAlpine, S.N. Chen, G.F. Pauli, Orthogonal analytical methods for botanical standardization:Determination of green tea catechins by qNMR and LC-MS/MS. J. Pharm. Biomed. Anal. 93 (2014) 59-67.

[38] T. Schoenberger, S. Menges, M.A. Bernstein, M. Pérez, F. Seoane, S. Sýkora, C. Cobas, Improving the Performance of High-Precision qNMR Measurements by a Double Integration Procedure in Practical Cases. Anal. Chem. (2016) 3836-3843.

[39] M. Tiainen, P. Soininen, R. Laatikainen, J. Magn. Reson. Quantitative Quantum Mechanical Spectral Analysis (qQMSA) of 1H NMR spectra of complex mixtures and biofluids 242 (2014) 67-78.

[40] P.M. Le, M. McCooeye, A. Windust, Characterization of the alkaloids in goldenseal (Hydrastis canadensis) root by high resolution Orbitrap LC-MS(n). Anal. Bioanal. Chem. 405 (2013) 4487-4498.

[41] A. Bornet, M. Maucourt, C. Deborde, D. Jacob, J. Milani, B. Vuichoud, X. Ji, J.-N. Dumez, A. Moing, G. Bodenhausen, S. Jannin, P. Giraudeau, Highly Repeatable Dissolution Dynamic Nuclear Polarization for Heteronuclear NMR Metabolomics. Anal. Chem. 88 (2016) 61796183.

[42] V. Daniele, F. Legrand, P. Berthault, J. Dumez, G. Huber, Single-Scan Multidimensional NMR Analysis of Mixtures at Sub-Millimolar Concentrations by using SABRE Hyperpolarization. Chemphyschem. 16 (2015) 3413-3417.

[43] B. Plainchont, P. Berruyer, J.-N. Dumez, S. Jannin, P. Giraudeau, Dynamic Nuclear Polarization Opens New Perspectives for NMR Spectroscopy in Analytical Chemistry. Anal. Chem. 90 (2018) 3639-3650. 